This et that dans les domaines spécialisés du corpus ICE-GB : quelles caractéristiques distributionnelles?

Thomas Gaillat

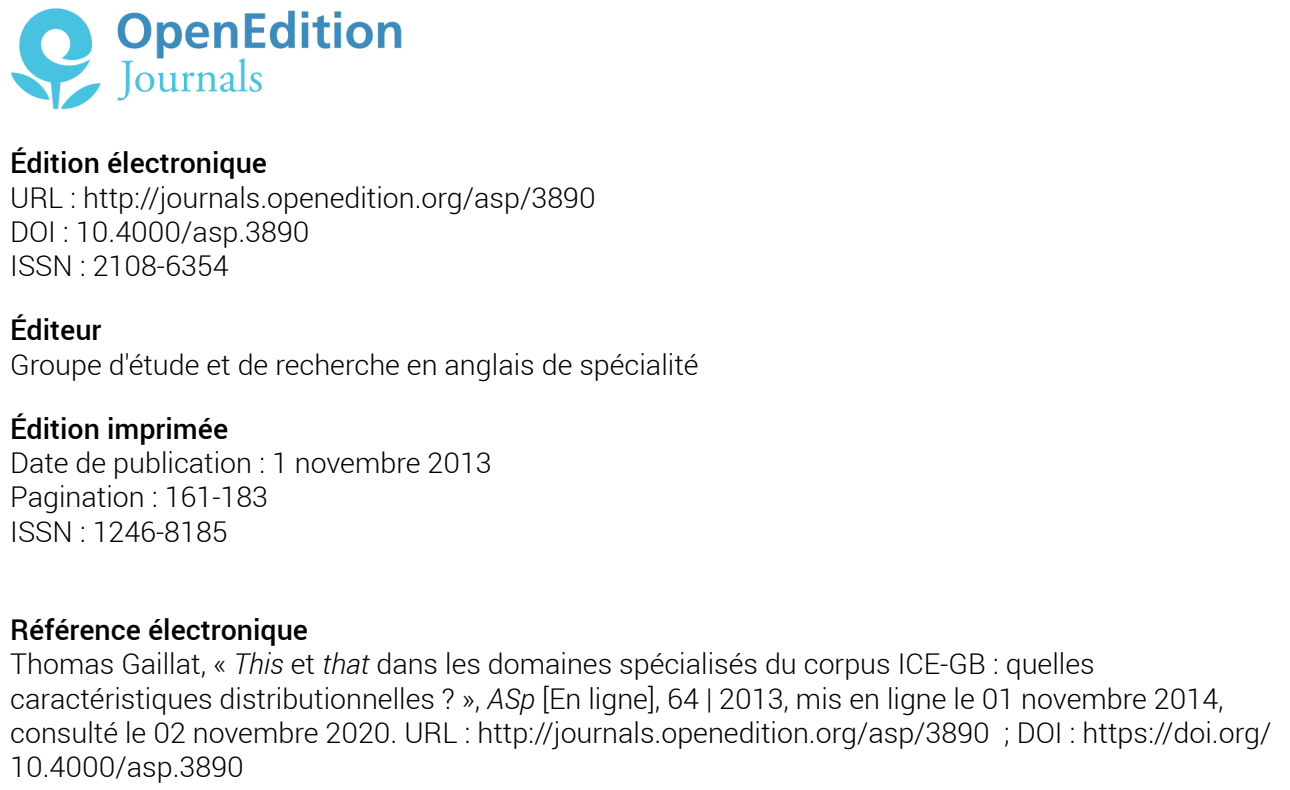

Ce document a été généré automatiquement le 2 novembre 2020.

Tous droits réservés 


\title{
This et that dans les domaines spécialisés du corpus ICE-GB : quelles caractéristiques distributionnelles?
}

\author{
Thomas Gaillat
}

\section{Introduction}

1 L'acte d'enseignement en anglais de spécialité soulève des questions dont certaines solutions trouvent leur source en didactique et en linguistique. Pour construire des dispositifs d'enseignement LANSAD, il est nécessaire de s'appuyer sur des connaissances linguistiques et culturelles propres au domaine de spécialité. Ces connaissances sont constituées à la fois d'éléments pédagogiques spécifiques tels que les conditions d'apprentissage et le type de public, mais aussi d'éléments linguistiques spécifiques des domaines spécialisés auxquels la spécialité enseignée se rattache. Nous nous rallions à Michel Petit (2010 : \$20) qui définit chaque domaine spécialisé comme

un secteur de la société constitué autour et en vue de l'exercice d'une activité principale qui, par sa nature, sa finalité et ses modalités particulières ainsi que par les compétences particulières qu'elle met en jeu chez ses acteurs, définit la place reconnaissable de ce secteur au sein de la société et d'un ensemble de ses autres secteurs et détermine sa composition et son organisation spécifiques.

Comprendre les éléments constitutifs du discours d'un domaine spécialisé constitue un enjeu important. Cette compréhension permet ainsi de nourrir, à la manière des études portant sur des variations linguistiques en fonction des genres discursifs (Hyland 2010), d'éventuelles applications didactiques dans l'enseignement de l'anglais de spécialité (Parkinson \& Adendorff 2004).

3 Notre expérience auprès d'étudiants LANSAD des domaines médical, scientifique et technologique nous a permis de constater la difficulté qu'éprouvent les apprenants à construire des textes cohésifs. Nous nous référons ici à Halliday et Hasan (1976), selon 
qui la cohésion d'un texte dépend, entre autres, du concept de référence, deux de ses constituants majeurs étant this et that. Une étude menée sur le corpus d'apprenants LONGDALE (Gaillat 2013) aborde la problématique de la construction de la référence chez les apprenants. Elle met en évidence des difficultés de choix entre les deux formes, mais aussi entre l'une ou l'autre des formes et le pronom personnel it ou l'article the. Afin de mieux appréhender l'usage fait par les apprenants des démonstratifs pour construire leur discours en langue de spécialité, il paraît nécessaire d'analyser, dans un premier temps, leur usage par les locuteurs natifs en anglais général et en fonction de certains domaines spécialisés (médecine, science et technologie). Fondée sur des données chiffrées, cette analyse fait apparaître des tendances d'utilisation de this et de that selon le domaine spécialisé. Les observations réalisées dans le cadre de cette étude auront peut-être à l'avenir des applications didactiques.

La recherche entreprise vise à dresser la carte des réalisations de this et de that en fonction de domaines spécialisés scientifiques. Pour ce faire, trois catégories de textes écrits et oraux ont été extraits du corpus International Corpus of English - Great Britain (ICE-GB): les textes d'anglais général, les textes du domaine médical et ceux du domaine scientifique et technologique. Pour la suite de l'article, et afin de favoriser la clarté de notre propos, nous utilisons le terme de sous-corpus pour désigner ces catégories, bien que cet usage soit quelque peu imprécis à propos de l'anglais général, car il s'agit du corpus entier. L'objet de cette recherche est d'identifier certaines tendances dans l'usage des démonstratifs, selon qu'ils sont utilisés dans un domaine spécialisé ou dans un autre. Nous focalisons notre propos sur les usages en pro-forme et déterminant, codés distinctement dans le corpus ICE-GB (Nelson, Wallis \& Aarts 1998). Cependant, nous élargissons notre analyse des données aux autres catégories grammaticales auxquelles peuvent appartenir les formes, à savoir les usages complétif et relatif de that d'une part, et l'usage adverbial des deux formes d'autre part.

Les travaux antérieurs abordent this et that selon deux perspectives. La première concerne les réalisations fonctionnelles des deux formes. Les positions syntaxiques qu'elles peuvent occuper sont doubles. On peut retrouver this/that devant un syntagme nominal ou en position de syntagme nominal. Les Anglophones utilisent les termes modifier pour la position ante-nominale et head pour la position nominale. Quirk, Leech et Svartvik (1985) distinguent usage en déterminant et usage nominal ; Fraser et Joly (1979) distinguent les pronoms supplétifs et les pronoms complétifs. Pour notre part, nous reprenons les termes de "déterminant » et de "pro-forme " (Lapaire \& Rotgé 2008: 50-51). La catégorie de "déterminant» permet d'affirmer la position antenominale des formes lorsqu'elles participent au processus de détermination d'un nom. «Pro-forme » permet d'indiquer les formes this/that qui apparaissent sans nom et qui jouent le rôle de syntagme nominal. En outre, la dénomination " pro-forme » éclaire sur le rôle de référence sémantique d'une forme qui peut être plus étendue qu'un groupe nominal isolé.

6 La seconde perspective concerne la fonction référentielle, plus particulièrement le domaine de la deixis. Traditionnellement, les deux formes permettent d'exprimer la distance qui sépare le locuteur de l'objet auquel il/elle fait référence. Cette valeur est reprise par Biber (1999 : 347) selon qui la distinction s'opère en fonction de la distance du référent. Une analyse plus approfondie permet de situer les deux formes en fonction du type de référence qu'elles mettent en œuvre. La distinction endophore/exophore est développée par Halliday et Hasan (1976) et reprise par Fraser et Joly (1979: 105) qui 
ajoutent la notion de sphère du locuteur comme repère. This place le référent dans la sphère du locuteur quand that le place en dehors. Lapaire et Rotgé (2008: 59) reprennent l'idée selon laquelle le «moi » énonciateur indique que le référent, désigné par le démonstratif, s'approche ou s'éloigne. Ils proposent l'idée de clôture avec that et de non-clôture avec this. This traduit le «refus ou impossibilité de traiter comme clos l'acquis opérationnel marqué par TH-». That dénote « l'acceptation, bouclage, ou, plus généralement, clôture de l'acquis opérationnel marqué par TH » (Lapaire \& Rotgé 2008 : 64). Le rôle de l'énonciateur est central dans leur explication. Huddleston et Pullum (2002 : 1504-1510) ${ }^{1}$ s'inscrivent, eux aussi, dans l'architecture exophore/endophore en attribuant des valeurs anaphorique ou déictique aux deux formes. L'anaphore reprend ce qui se trouve dans le co-texte, quand le déictique renvoie à des objets présents dans la situation d'énonciation. Ce type de reprise situationnelle tend à favoriser la valeur de proximité ou d'éloignement dans le processus de référence et à ne pas prendre en compte l'énonciateur comme responsable des opérations cognitives construisant la référence, ce qui est central chez les auteurs francophones mentionnés (Lapaire \& Rotgé ; Fraser \& Joly ; Cotte). Kleiber (1992), quant à lui, différencie le déictique de l'anaphorique, selon qu'il s'agit d'information nouvelle ou non (et non plus en fonction de la localisation situationnelle ou contextuelle). Cotte (1993: 47) réconcilie les deux points de vue en montrant que ce qui est déjà présent pour le locuteur peut être perçu comme «nouveau dans le rapport interlocutif » et, donc, nouveau pour l'allocutaire : il y a une valeur de reprise anaphorique si l'on se place du point de vue du locuteur et une valeur déictique si l'on se place du point de vue de l'allocutaire. Bordet (2011) relève cette ambivalence dans son travail sur this lorsqu'elle indique sa capacité à faire progresser la focalisation tout en assurant l'établissement de la référence. Ce faisant, elle rejoint ainsi Cornish (1999: 31-32) qui considère les procédures déictiques et anaphoriques comme des fonctions du discours « constituant un continuum sur le plan de l'indexicalité ». Dans la suite de cet article, nous nous référons aux deux formes en utilisant le terme historique, et peut-être plus neutre, de "démonstratifs ». Nous réservons les termes « anaphorique » et « déictique » pour les explications relatives au fonctionnement référentiel des démonstratifs.

7 Après la catégorisation du corpus ICE-GB, des relevés ont été effectués pour déterminer les fréquences d'occurrence de this et de that, dans le cadre de notre projet, qui consiste à identifier les corrélations qui existent entre les domaines spécialisés de discours et ces marqueurs. La première partie de l'article est consacrée aux données, à leur collecte et à leur normalisation. Dans un deuxième temps, nous menons une analyse statistique sur les profils de corpus en fonction des différentes catégories grammaticales de this et de that. Nous tentons d'identifier le rôle que peut jouer un this ou un that en fonction d'une catégorie donnée et des domaines spécialisés. L'évaluation statistique de la distribution de this et de that est ensuite mise en œuvre. La troisième partie de l'article propose un retour sur les données et analyse la distribution textuelle de this et de that au sein de textes médicaux écrits et oraux. Elle permet de vérifier les observations effectuées à l'issue de l'analyse statistique et de définir les bases de ce qui paraît être la prochaine étape de la linguistique textuelle: caractériser les étapes obligées des structures textuelles par leurs marqueurs de prédilection. De ce point de vue, l'anglais de spécialité, en particulier dans des écrits extrêmement codés tels que les résumés ou les articles scientifiques, constitue un observatoire privilégié. 


\section{Méthodologie}

8 Il s'agit ici de décrire le corpus ICE-GB et la structuration des données le constituant. Est présentée ensuite la manière dont sont extraits et catégorisés en sous-corpus les échantillons comprenant this et that.

\subsection{Description du corpus ICE-GB}

9 Le corpus ICE-GB est composé de 1061263 mots. Aarts, Nelson et Wallis (2007) précisent qu'il comprend 500 textes d'environ 2000 mots chacun, qui proviennent de la langue orale ou écrite et qui ont été collectés entre 1990 et 1996 : leurs auteurs étaient alors âgés de 18 ans et plus. Ils ont été catégorisés de manière à offrir une traçabilité du type de texte. Par exemple, un certain nombre d'entre eux, utilisés dans la présente étude, sont classés selon le chemin suivant: Spoken $>$ Dialogues $>$ Public > Classroom lessons. Chaque texte possède une fiche caractéristique précise sur son auteur, son lieu et date de collecte et sa thématique de contenu. Un large éventail de catégories caractérise ces textes, qu'il s'agisse du mode oral ou écrit. À l'oral, on trouve des dialogues enregistrés lors de conversations directes ou téléphoniques, ou lors d'émissions de radio, ou encore lors de séances parlementaires. Le corpus oral comprend aussi, entre autres, des monologues enregistrés lors de conférences ou de leçons en classe. Le mode écrit comporte des textes universitaires et scientifiques dans les domaines des sciences humaines, des sciences sociales et des technologies. Le corpus comprend des écrits journalistiques, administratifs, ou encore littéraires (extraits de romans). Afin d'explorer le corpus, le logiciel ICECUP ${ }^{2}$ a été développé et propose plusieurs outils, dont un outil de navigation appelé Corpus map. Il permet d'évoluer dans les catégories et sous-catégories à la manière d'un explorateur de fichiers (Nelson, Wallis \& Aarts 2002 : 87). En parcourant l'ensemble des textes classés dans la catégorie Classroom lessons, par exemple, quatre leçons au contenu médical totalisant 8249 mots sont identifiées.

Outre une classification thématique des textes, le corpus ICE-GB propose une annotation syntaxique de ceux-ci, sous forme d'arbres permettant d'effectuer des requêtes à l'aide d'un autre outil du logiciel ICECUP. Avant d'aborder les requêtes, il est important d'expliquer la structuration de l'annotation syntaxique et des parties du discours élaborée par les auteurs du corpus ICE-GB. La figure 1 (Nelson, Wallis \& Aarts 1998) décrit la manière dont se structure l'annotation «partie-du-discours » sous forme de «nœuds». Une nomenclature précise est utilisée pour décrire chaque unité ${ }^{3}$. Un premier élément identifie la fonction (par exemple, Phrase Unit, Adverb Head, Noun Phrase Head, Subordinate Head), c'est-à-dire le rôle que joue chaque mot par rapport à son contexte immédiat. Sa catégorie grammaticale est ensuite spécifiée. Par exemple, this et that pourront se voir assigner les étiquettes PRON pour pronoun ou DTCE pour Central Determiner. Le troisième élément concerne des caractéristiques particulières que peuvent prendre les formes. Concernant this et that, il s'agira par exemple de l'étiquette DEM pour démonstratifs. Chacun de ces nœuds s'articule avec les autres, de telle manière qu'une représentation syntaxique par arborescence est possible. Cette arborescence résulte d'une analyse automatique faite par un parser, développé à l'Université de Nijmegen, qui utilise une grammaire formelle pour analyser chaque 
unité au niveau des mots, des groupes de mots et des propositions (Aarts, Nelson \& Wallis $2007: 29$ ).

Figure 1 Annotation « partie-du-discours » du corpus ICE-GB

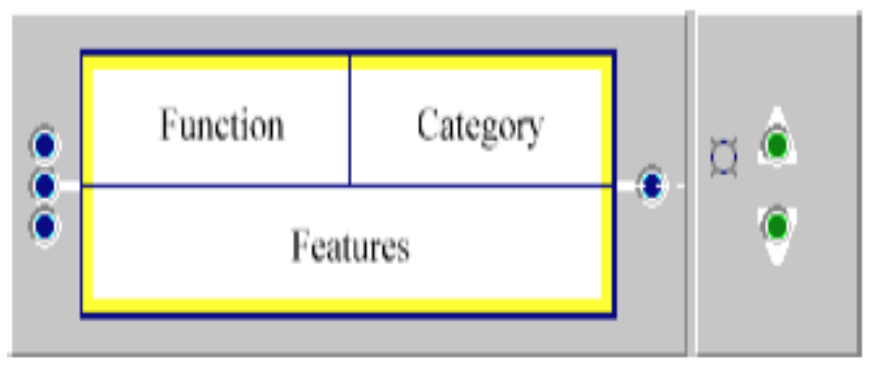

11 Abordons maintenant la question des requêtes utiles pour l'extraction des occurrences de this et de that. La structuration des données, sous forme d'annotation syntaxique, sert de point de départ pour élaborer des requêtes sur le corpus. Il s'agit de convertir les catégories grammaticales, sur lesquelles l'utilisateur souhaite faire une recherche, en requêtes logiques compatibles avec la structuration des données du corpus ICE-GB. Les Fuzzy Tree Fragments ou FTF, outils de recherche par motif du logiciel ICECUP, permettent de modéliser chaque requête de façon intuitive. Leur principe de fonctionnement repose sur la structuration des nœuds et sur leurs articulations. Plutôt que d'utiliser le langage Logic, d'un abord complexe, l'utilisateur construit un motif du type du fragment d'arbre syntaxique qu'il souhaite trouver. Les liens plus ou moins stricts de domination et d'adjacence entre les nœuds sont définis et les catégories, fonctions et caractéristiques de chaque nœud sont renseignées partiellement ou de manière exhaustive. Des mots peuvent être ajoutés au motif FTF qui traduit la requête en langage Logic. Après validation, toutes les occurrences dont les mots et l'annotation correspondent au motif seront affichées avec leur contexte proche. La section 2.2 aborde la syntaxe exacte des requêtes élaborées pour les besoins de l'étude.

\subsection{Extraction et catégorisation en sous-corpus}

12 À présent que le corpus ICE-GB, sa structuration et ses outils ont été exposés, il s'agit de décrire la première étape de cette étude. Elle consiste à subdiviser le corpus en trois sous-corpus sur lesquels seront effectuées des requêtes d'ordre syntaxique qui permettront d'extraire les occurrences de formes en fonction de schémas syntaxiques. Le premier niveau de division concerne les modalités orales ou écrites. Pour chacun de ces deux ensembles, nous distinguons trois types de séries de textes. La première concerne les textes dont les contenus sont médicaux. La deuxième série concerne les textes scientifiques et technologiques (textes médicaux exclus) et la troisième série correspond à tous les textes du corpus ICE-GB (y compris ceux des deux premières séries), et s'inscrit donc dans le domaine de l'anglais général. Chaque série est identifiée à partir des données sociolinguistiques (discipline de l'auteur, institution) fournies pour chacun des textes. Il y a au final trois sous-corpus pour chaque mode oral ou écrit, soit un total de six sous-corpus.

13 L'outil d'exploration ne permet pas de procéder à des regroupements ad hoc qui autoriseraient une seule manipulation pour la sélection de l'ensemble des textes correspondant à un contenu particulier. L'outil Corpus map permet d'identifier 
l'ensemble des textes scientifiques du corpus et leur nombre de mots. Chaque texte est trié manuellement en fonction du domaine scientifique dont il relève. Ce processus permet, par addition, d'obtenir le nombre d'occurrences de mots par catégorie (tableau 1). Concernant l'origine des quatre sous-corpus médicaux, scientifiques et technologiques, ceux-ci comprennent des textes classés dans les catégories suivantes: leçons (classroom lessons), monologue, démonstrations scientifiques non rédigées (unscripted demonstrations) et monologue, discours non rédigés (unscripted speeches) pour le mode oral; écrits scientifiques et universitaires (academic writing) et écrits non scientifiques (non-academic writing), chaque catégorie du mode écrit incluant les souscatégories « sciences naturelles » et « technologie » (natural sciences and technology).

Tableau 1 Ensemble des sous-corpus extraits du corpus ICE-GB

\begin{tabular}{|l|c|}
\hline \multicolumn{1}{|c|}{ Discours } & $\begin{array}{c}\text { Tailles des } \\
\text { sous-corpus }\end{array}$ \\
\hline Oral médical & 15178 \\
\hline Oral- sci \& tech (sans méd) & 20680 \\
\hline Oral général & 637682 \\
\hline Écrit médical & 8431 \\
\hline Ecrit sci \& tech (sans méd) & 38202 \\
\hline Écrit général & 423581 \\
\hline
\end{tabular}

14 Une fois ces catégories établies, la deuxième étape de l'étude consiste à élaborer des requêtes pour faire le décompte du nombre d'occurrences de chaque démonstratif singulier et pluriel en fonction de sa catégorie grammaticale. Il s'agit de formaliser de manière logique des requêtes grâce à l'outil du logiciel ICECUP (Nelson, Wallis \& Aarts 2002). Ce travail se situe dans le prolongement d'une étape préliminaire d'identification des catégories grammaticales auxquelles peuvent correspondre ces deux formes. Les travaux de recherche sur le sujet, présentés dans l'introduction de cet article, permettent d'avancer cinq catégories grammaticales possibles pour that et trois pour this. Elles sont résumées dans le tableau 2, avec des exemples tirés du corpus ICE-GB. Bien que de nombreux auteurs aient glosé sur la dimension sémantique prise par les deux formes, notamment en ce qui concerne le domaine de la deixis (cf. introduction), cette étude se concentre sur leur rôle syntaxique, en relation avec l'annotation du corpus. 
Tableau 2 Les différentes catégories grammaticales de this et de that

\begin{tabular}{|l|l|l|l|}
\hline Forme & $\begin{array}{c}\text { Catégorie } \\
\text { grammaticale }\end{array}$ & \multicolumn{1}{|c|}{ Définiton } & \multicolumn{1}{|c|}{ Exemple } \\
\hline This & pro-forme & $\begin{array}{l}\text { Remplace une forme telle que prédicat, proposition, } \\
\text { phrase,conglomérat d'énoncés, nom }\end{array}$ & $\begin{array}{l}\text { This is not like a real situation (SIB-004-136) } \\
\text { (Cela ne ressemble pas à une vrai situation) }\end{array}$ \\
\hline This & adverbial & Modifie un adjectif & $\begin{array}{l}\text { When you felt this strong [...] (W2F-008- } \\
\text { O38) (Quand tu t'es senti aussi fort que ça) }\end{array}$ \\
\hline This & déterminant & Introduit un syntagme nominal & $\begin{array}{l}\text { From the actual target tissue which in this } \\
\text { particular case we are talking about is musle } \\
\text { [...] (S1B-009-192) (à partir du tissu cible } \\
\text { qui dans le cas particulier dont nous parlons } \\
\text { est musculaire) }\end{array}$ \\
\hline That & pro-forme & $\begin{array}{l}\text { Remplace une forme telle que prédicat, proposition, phrase, } \\
\text { conglomérat d'énoncès, nom }\end{array}$ & $\begin{array}{l}\text { That sounds reasonable (S1B-003-019) } \\
\text { (Cela semble raisonnable) }\end{array}$ \\
\hline That & déterminant & Introduit un syntagme nominal & $\begin{array}{l}\text { and yet that language was being channelled } \\
\text { into a very narrow, field (S1A-001) (et } \\
\text { pourtant ce langage [corporel] était véhiculé } \\
\text { dans un domaine très spécifique) }\end{array}$ \\
\hline That & adverbial & Modifie un adjectif & $\begin{array}{l}\text { And it's not that far anyway (S1A-006-294) } \\
\text { (Et ce n'est pas si loin) }\end{array}$ \\
\hline That & conjonctif & Introduit une subordonnée, complément d' un verbe & $\begin{array}{l}\text { It means that the zero level is variable so } \\
\text { that you get readings [...] (S1B-004-252) } \\
\text { (Cela signifie que le niveau zéro est variable } \\
\text { afin de pouvoir obtenir des indications) }\end{array}$ \\
\hline That & relatif & $\begin{array}{l}\text { But it's random variation as we said before } \\
\text { that will tend to even out[...] (S1B-004-346) } \\
\text { (Mais ce sont des variations aléatoires } \\
\text { comme on l'a déjà dit qui vont aller en } \\
\text { s'aplanissant...) }\end{array}$ \\
\hline
\end{tabular}

Les requêtes sont élaborées à l'aide de l'outil Logic de ICECUP. Par exemple, afin d'identifier les occurrences de this pro-forme, nous décrivons le motif correspondant à toutes les occurrences du mot this en position de tête de syntagme nominal (on ne renseigne que la zone Function avec NPHD pour Noun Phrase Head). Traduite en langage Logic (Aarts, Nelson \& Wallis $2007: 30$ ), la requête prend la forme this $+<$ NPHD> et suffit à isoler tous les this pro-formes ayant pour catégorie "pronom» (pron) et pour caractéristique « démonstratif» (dem). Plus simplement, la requête this $+<\mathrm{DTCE}>$ donne l'ensemble des formes de this déterminant. Concernant that, et du fait de ses cinq catégories grammaticales possibles, certaines requêtes sont plus détaillées, notamment celles correspondant au that pro-forme. La requête that $<$ NPHD> couvre les formes "démonstratif» et "pronom relatif». S'il s'agit d'isoler l'une de l'autre, les deux requêtes suivantes doivent être saisies: that $+<\mathrm{NPHD}, \mathrm{PRON}(\mathrm{dem})>$ et that $t<\mathrm{NPHD}$, PRON(rel)>. C'est donc grâce à l'utilitaire de recherche du logiciel, associé à un langage de requête simple, qu'il est possible d'extraire l'ensemble des formes et d'obtenir des fenêtres de résultats les présentant en contexte.

La collecte des occurrences de this et de that peut donc s'effectuer texte par texte, et permet d'obtenir un tableau synthétique des fréquences absolues par sous-corpus. Du fait de l'irrégularité de taille de chaque sous-corpus, il convient, avant toute analyse, de normaliser ces résultats en suivant Gries (2010). Afin de prendre en compte la différenciation oral/écrit par domaine spécialisé, essentielle à l'analyse, il est nécessaire de normaliser les fréquences au regard de chacun des sous-corpus oral et écrit du corpus ICE-GB. Par exemple, le sous-corpus oral médical comprend 15178 mots et le sous-corpus écrit en totalise 8 431. Cette normalisation permet de produire les fréquences relatives par million de mots pour chaque occurrence et pour chaque catégorie grammaticale, à la manière des deux exemples suivants :

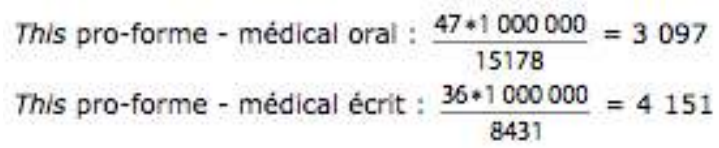

Une fois effectué ce travail de normalisation, le travail d'analyse des données commence pour déterminer les différences entre les corpus et, donc, entre les 
domaines spécialisés et l'anglais général, mais aussi entre les catégories grammaticales des deux formes.

\section{Analyse et résultats}

Les données collectées et normalisées sont consignées dans un tableau synthétique permettant de répondre à plusieurs questions. La première consiste à s'interroger sur les différences qui existent entre les deux formes sans tenir compte de leur catégorie grammaticale. La deuxième porte sur la mise en regard des sous-corpus en fonction des formes et de leur catégorie grammaticale, en prenant également en compte les différences comparatives. Une dernière question concerne la validité des observations par rapport à la loi du $\chi^{2}$. Les variations des données analysées suivent-elles cette loi ou sont-elles dues à des facteurs propres aux catégories choisies ? Le test du $\chi^{2}$ permet de signaler une corrélation entre les catégories de données.

\subsection{Approche globale}

L'approche globale consiste à prendre en compte l'ensemble des formes de this et de that et à observer leur distribution sans distinction, c'est-à-dire sans tenir compte de leurs catégories grammaticales particulières. Il s'agit des parties grisées du tableau 3 (a et b). Celui-ci regroupe l'ensemble des données relatives aux usages de this et de that selon leur catégorie grammaticale et selon le sous-corpus.

Tableau 3a Données normalisées : fréquences relatives observées dans les parties orale et écrite du corpus ICE-GB par million de mots

\begin{tabular}{|l|c|c|c|c|}
\hline \multicolumn{1}{|c|}{ Discours } & This_tous & $\begin{array}{c}\text { this pro- } \\
\text { forme }\end{array}$ & $\begin{array}{c}\text { this } \\
\text { déterminant }\end{array}$ & this adverbial \\
\hline Oral médical & 9883 & 3097 & 6786 & 0 \\
\hline Oral sci \& tech (sans méd) & 13201 & 4932 & 8269 & 0 \\
\hline Oral général & 7259 & 2744 & 4509 & 6 \\
\hline Ecrit médical & 9252 & 4151 & 5100 & 0 \\
\hline Ecrit sci \& tech (sans méd) & 8874 & 3586 & 5288 & 0 \\
\hline Ecrit général & 7120 & 2828 & 4287 & 5 \\
\hline
\end{tabular}

Tableau 3b Données normalisées : fréquences relatives observées dans les parties orale et écrite du corpus ICE-GB par million de mots

\begin{tabular}{|l|c|c|c|c|c|c|}
\hline \multicolumn{1}{|c|}{ Discours } & that_tous & $\begin{array}{c}\text { that pro- } \\
\text { form }\end{array}$ & $\begin{array}{c}\text { that } \\
\text { Déterminant }\end{array}$ & that adverbial & $\begin{array}{c}\text { that } \\
\text { complementif }\end{array}$ & that pron rel \\
\hline Oral médical & 25827 & 8301 & 4151 & 2570 & 5996 & 4810 \\
\hline Oral sci \& tech (sans méd) & 23743 & 6867 & 3385 & 2466 & 5319 & 5706 \\
\hline Oral général & 23803 & 7761 & 2895 & 2275 & 6768 & 4104 \\
\hline Ecrit médical & 12573 & 2728 & 830 & 0 & 5337 & 3677 \\
\hline Ecrit sci \& tech (sans méd) & 8638 & 1361 & 1021 & 0 & 4607 & 1649 \\
\hline Ecrit général & 10699 & 1598 & 958 & 19 & 6209 & 1915 \\
\hline
\end{tabular}

Pour un premier niveau d'analyse, le calcul des fréquences relatives totales de this et de that observées dans les parties orale et écrite du corpus ICE-GB est effectué (cf. figure 2). 
Figure 2 Fréquences relatives de this dans le corpus ICE-GB

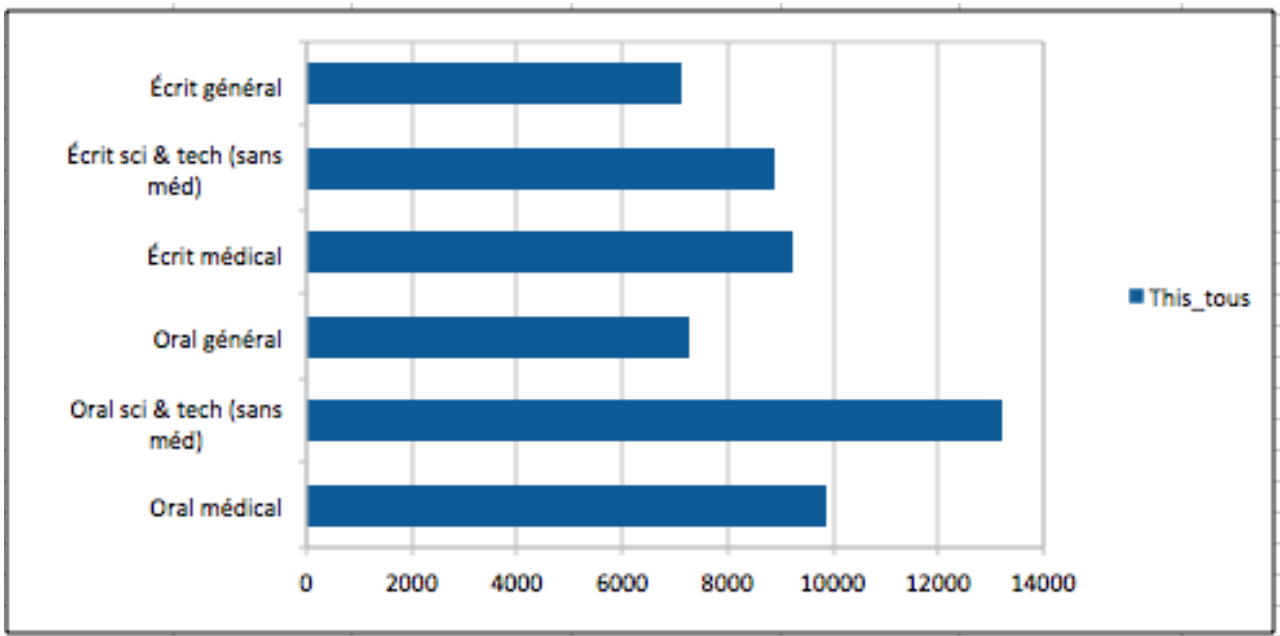

21 On observe clairement une équivalence des usages au sein de chaque mode oral ou écrit, excepté dans les sous-corpus scientifiques et technologiques qui présentent des fréquences d'utilisation plus élevées. Celles-ci peuvent s'expliquer par une hétérogénéité thématique relative à l'intérieur de ces deux sous-corpus, l'ensemble oral comportant peu de textes technologiques et une prépondérance de textes proches des sciences naturelles. À l'inverse, le sous-corpus écrit comprend une part importante de textes technologiques. Par conséquent, il est possible que les textes technologiques comportent une variation que l'on retrouve dans cet histogramme. Si l'on compare simplement les sous-corpus médical et général, on trouve un usage équivalent de this entre oral et écrit.

Figure 3 Fréquences relatives de that dans le corpus ICE-GB

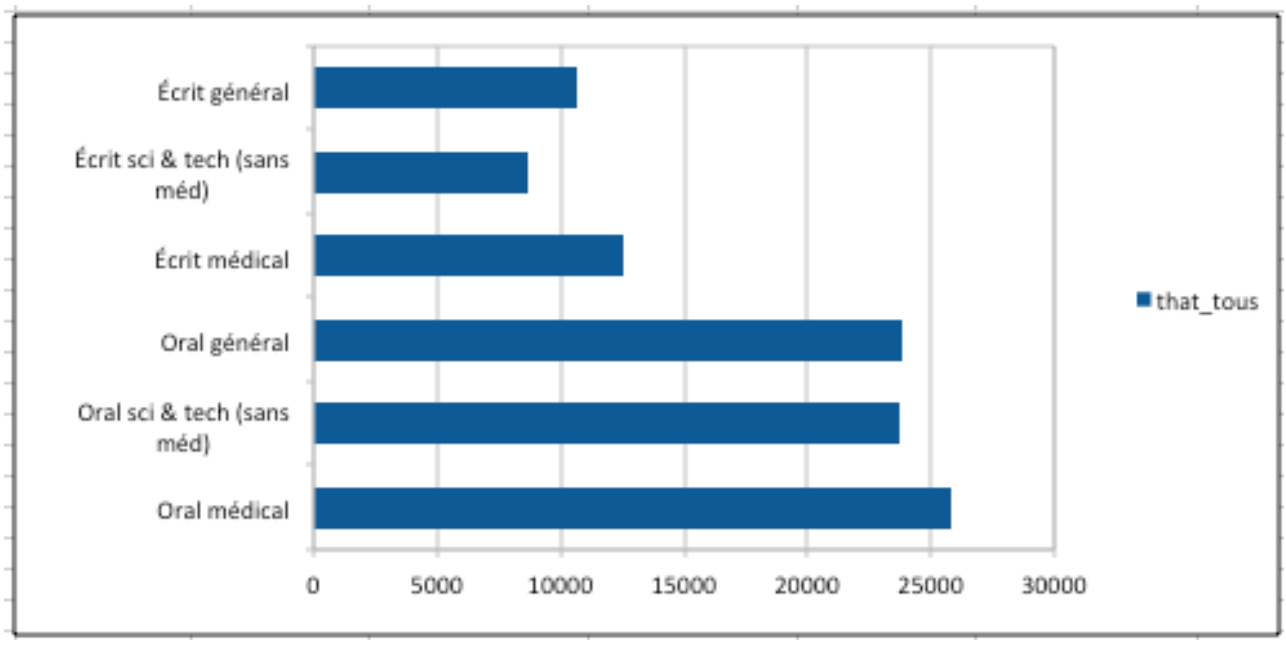

Les observations de that en fonction des différents sous-corpus (cf. figure 3) révèlent des différences notables entre chaque mode oral ou écrit. That est systématiquement sous-utilisé à l'écrit par rapport à l'oral. Il y a donc deux tendances inverses entre this et that. Si l'on considère les différentes catégories grammaticales des deux formes, on voit que that recouvre les mêmes catégories que this (pro-forme, déterminant, adverbial), mais en couvre d'autres très importantes à l'oral, comme la forme complétive que l'on retrouve dans les énoncés de type I think that... et la forme pronom 
relatif. En termes schématiques, this permet seulement la reprise d'éléments du discours, quand that va au-delà, en permettant la complexification du discours grâce à des structures syntaxiques fondées sur l'hypotaxe, prépondérante à l'écrit.

\subsection{Approche détaillée}

En reprenant les résultats détaillés du tableau 3 et en les comparant avec les résultats obtenus par Biber (Biber et alii 1999 : 274) et publiés dans Longman Grammar of Spoken and Written English (LGSWE), on peut faire les observations suivantes concernant l'usage en déterminant des formes (cf. tableau 4). On prendra soin de noter que les relevés de Biber concernent des conversations quand ceux du corpus ICE-GB comprennent aussi des monologues. Là où Biber et alii relèvent une fréquence de 2500 this par million de mots à l'oral (singulier et pluriel) en conversation, le relevé dans le corpus ICE-GB en comprend 4500 dans le domaine de l'anglais général à l'oral. En ce qui concerne les occurrences de that (singulier et pluriel) en fonctionnement déterminant, le corpus ICEGB donne une fréquence de 2900 contre 2500 dans l'étude de Biber et alii. Le comparatif des usages en pro-forme donne les observations suivantes. Biber et alii (1999: 349) rapportent une fréquence de 2000 this (singulier et pluriel) en conversation. Le relevé dans le corpus ICE-GB en donne 2700 dans le domaine de l'anglais général à l'oral. That (singulier et pluriel) en conversation totalise une fréquence de 11500 dans l'étude de Biber et alii, quand le relevé du corpus ICE-GB en montre 7800 dans le domaine de l'anglais général à l'oral. En résumé, le comparatif des pro-formes montre plus de that dans l'étude de Biber et alii que dans le corpus ICE-GB et moins de this que dans le corpus ICE-GB. Au niveau des déterminants, le corpus ICE-GB donne plus de this que la LGSWE et une fréquence similaire pour that.

Tableau 4 Comparatif des formes entre le corpus de Biber et le corpus ICE-GB

\begin{tabular}{|l|c|c|}
\hline & Biber & ICE-GB \\
\hline This (déterminant) & 2500 & 4500 \\
\hline That (déterminant) & 2500 & 2900 \\
\hline This (pro-forme) & 2000 & 2700 \\
\hline That (pro-forme) & 11500 & 7800 \\
\hline
\end{tabular}

Le domaine de l'anglais général à l'oral général du corpus ICE-GB inclut non seulement des conversations, mais aussi des monologues et des leçons, ce qui peut expliquer cette différence. En effet, le monologue, auquel s'apparente aussi la leçon, a souvent pour objectif l'explication d'un concept en situation, ce qui peut impliquer un rapprochement dudit concept vers la sphère de l'énonciateur de manière physique ou mémorielle. Ceci est illustré dans l'exemple identifié S2A-051: You'll see creatures like this one and if you try and capture it you can spend ages and ages watching them. Il s'agit d'une leçon de biologie et le locuteur focalise son propos sur le terme creatures, élément appartenant à son environnement, ce qui implique l'utilisation de this. Le monologue correspond lui aussi à une construction de discours dont la cohésion dépend de l'environnement textuel et physique organisé par l'auteur du discours. Il en découle un grand nombre de référents étroitement liés au locuteur de manière déictique ou anaphorique, d'où l'usage de this. 
rsement, le fait que that soit moins fréquent dans le corpus ICE-GB que dans la LGSWE peut aussi provenir de la part importante des monologues et des leçons dans le corpus. La conversation implique un co-locuteur. Contrairement au monologue, où le locuteur fait référence à des concepts qu'il fait siens, la conversation implique des reprises de concepts portés par le co-locuteur. Ces reprises signalent probablement, le plus souvent, une acceptation sans modification des caractéristiques du référent, ce que that est apte à faire. L'exemple suivant, extrait (S1A-012) des conversations directes du corpus, illustre le rôle joué par that dans un dialogue.

Locuteur C: «I ' $m$ not really in favour of boys' schools taking on girls in the sixth

form and that 's it. It would be a very odd compromise »

Locuteur D : «Uhm yes »

Locuteur $\mathrm{E}:$ « Uh uhm »

Locuteur C : «Added to which of course it destroys girls' schools »

Locuteur B : « Yes it certainly does that »

La première occurrence de that permet au locuteur d'affirmer son point de vue et de clore le débat et la seconde permet la reprise par le locuteur B de l'argument avancé par le locuteur C. Selon Lapaire et Rotgé, il y a «acceptation, bouclage, ou, plus généralement, clôture (-AT) de l'acquis opérationnel marqué par TH » (2008:64) avec that. Le fait que le corpus ICE-GB contienne une grande part de monologues tend à réduire la part relative des conversations au regard du corpus de Biber et alii. Ceci peut expliquer la relative infériorité numérique des occurrences de that dans le corpus ICEGB.

Considérant les deux tendances de this et de that, et afin d'affiner les rôles que les deux formes jouent, il est nécessaire de procéder à une analyse plus détaillée des sous-corpus en fonction des catégories grammaticales. Le calcul des profils par colonne et par ligne du tableau 3 donne les résultats suivants.

Tableau 5 Profils par colonne : importance relative des sous-corpus pour chaque catégorie grammaticale (pourcentage)

\begin{tabular}{|c|c|c|c|c|c|c|c|c|}
\hline Discours & $\begin{array}{l}\text { this pro } \\
\text { forme } \\
\text { for }\end{array}$ & & & & & & & \\
\hline médical & & $\frac{20}{20}$ & . & 29 & 31 & 35 & 18 & 22 \\
\hline $\begin{array}{l}\text { al sci \& tech (sans méd) } \\
\text { al général }\end{array}$ & $\frac{23}{13}$ & $\frac{24}{13}$ & $\frac{0}{57}$ & $\begin{array}{r}24 \\
27 \\
\end{array}$ & $\begin{array}{l}26 \\
22 \\
\end{array}$ & $\begin{array}{r}34 \\
31 \\
\end{array}$ & $\frac{16}{20}$ & $\begin{array}{l}26 \\
19 \\
\end{array}$ \\
\hline crit médical & 19 & $\frac{15}{115}$ & 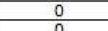 & 10 & 6 & 0 & 16 & 17 \\
\hline $\begin{array}{l}\text { Ecrit sci \& tec } \\
\text { Ecrit général }\end{array}$ & $\frac{17}{13}$ & $\frac{15}{13^{2}}$ & $\frac{0}{43}$ & $\frac{5}{6}$ & & $\frac{0}{0}$ & $\frac{13}{18}$ & $\frac{8}{9}$ \\
\hline
\end{tabular}

Les profils par colonne (tableau 5) permettent de comparer les sous-corpus entre eux, pour une forme donnée, et de voir si certaines catégories grammaticales sont plus fréquentes dans un sous-corpus que dans un autre. En d'autres termes, on mesure l'attractivité qu'exerce un sous-corpus par rapport aux autres, pour une occurrence de this ou de that d'une catégorie grammaticale donnée. Par exemple, on peut noter que le l'emploi de that qui correspond aux fonctions pro-forme et déterminant est plus fréquent dans les trois sous-corpus oraux que dans les sous-corpus écrits. Les souscorpus oraux sont donc plus attractifs vis-à-vis des that pro-forme et déterminant que les sous-corpus écrits. Cette observation corrobore celle de Biber et alii qui notent que la distribution des déterminants et des pronoms démonstratifs est plus importante dans le registre de la conversation qu'à l'écrit (Biber et alii 1999: 274, 349). Ceci peut s'expliquer par le fait que that est un outil anaphorique qui permet au locuteur la reprise de concepts déjà mentionnés (dans son discours). Le mode de l'oral impose une charge cognitive plus importante quant à la mémorisation des différents référents du 
discours. Par conséquent, l'utilisation de that permet une réactivation des référents en fonction des énoncés dans lesquels ils apparaissent.

Le cas de this répond à deux variations. En fonctionnement déterminant, this est plus présent à l'oral et, notamment, dans les sous-corpus scientifique, technologique et médical. En fonctionnement pro-forme, this est autant présent à l'oral qu'à l'écrit, notamment dans le sous-corpus scientifique et technologique et dans le sous-corpus médical. La langue scientifique a pour objectif l'observation, la démonstration et la leçon. Ce type de déroulement discursif favorise donc la mise au premier plan de concepts déjà exprimés dans le discours ou déjà connus. Le locuteur focalise son propos sur une entité pour développer son raisonnement en ajoutant de nouvelles informations sur le concept posé en amont. Shirley Carter-Thomas et Elisabeth RowleyJolivet (2001) soulignent que la dimension déictique de this sert à pointer vers un nouvel élément dans les présentations scientifiques à l'oral. Le référent porte de nouvelles informations et se trouve dans la sphère du locuteur (Fraser \& Joly 1979), d'où l'utilisation de this. Ce référent étant en cours d'analyse, il y a « impossibilité de traiter comme clos l'acquis opérationnel marqué par TH-» (Lapaire \& Rotgé 1998 : 63). La réalisation fonctionnelle en pro-forme, qui est assez fréquente à l'écrit, peut s'expliquer par la nécessité de reprise, sans pour autant nommer le concept une nouvelle fois, celui-ci ayant déjà été construit dans le co-texte. On a donc une focalisation sur un concept qui ne requiert pas de répétition de la dénomination.

Tableau 6 Profils par ligne: pourcentage des fréquences relatives de this et de that pour chaque sous-corpus

\begin{tabular}{|l|c|c|c|c|c|c|c|c|}
\hline \multicolumn{1}{|c|}{ Discours } & $\begin{array}{c}\text { this pro- } \\
\text { forme }\end{array}$ & $\begin{array}{c}\text { this } \\
\text { déterminant }\end{array}$ & $\begin{array}{c}\text { this } \\
\text { adverbial }\end{array}$ & $\begin{array}{c}\text { that pro- } \\
\text { forme }\end{array}$ & $\begin{array}{c}\text { that } \\
\text { déterminant }\end{array}$ & $\begin{array}{c}\text { that } \\
\text { adverbial }\end{array}$ & $\begin{array}{c}\text { that } \\
\text { complétif }\end{array}$ & $\begin{array}{c}\text { that pron } \\
\text { rel }\end{array}$ \\
\hline Oral médical & 9 & 19 & 0 & 23 & 12 & 7 & 17 & 13 \\
\hline Oral sci \& tech (sans méd) & 13 & 22 & 0 & 19 & 9 & 7 & 14 & 15 \\
\hline Oral général & 9 & 15 & 0 & 25 & 9 & 7 & 22 & 13 \\
\hline Ecrit médical & 19 & 23 & 0 & 13 & 4 & 0 & 24 & 17 \\
\hline Ecrit sci \& tech (sans méd) & 20 & 30 & 0 & 8 & 6 & 0 & 26 & 9 \\
\hline Ecrit général & 16 & 24 & 0 & 9 & 5 & 0 & 35 & 11 \\
\hline
\end{tabular}

Les profils par ligne (tableau 6) permettent de mesurer la part que prend une forme par rapport aux autres au sein d'un même sous-corpus. On peut, pour un sous-corpus, comparer l'importance donnée à chaque forme et à sa catégorie grammaticale. On observe de nettes variations au sein de chaque corpus. La mise en regard des souscorpus permet de comparer ces variations d'utilisation. Par exemple, dans le souscorpus oral médical, $9 \%$ des this sont des this pro-forme, alors que ce ratio est de $19 \%$ à l'écrit dans le domaine médical. On voit que this déterminant y est largement plus utilisé. Lors de l'analyse globale, les fréquences relatives de this étaient similaires entre oral et écrit. Mis en regard avec celles des autres formes pour un sous-corpus donné, on note que le poids relatif de this diffère. On observe en effet que, si on compte autant de this à l'écrit qu'à l'oral en anglais médical, le sous-corpus oral de ce domaine montre une sous-utilisation de this pro-forme par rapport à l'usage de toutes les autres formes de this et de that. Cette tendance est identique pour chaque sous-corpus oral. Inversement, this pro-forme joue un rôle majeur à l'écrit, ce qui rééquilibre la statistique globale. En ce qui concerne la forme that, sa part d'utilisation varie aussi selon les sous-corpus. That pro-forme joue un rôle significatif à l'oral, quel que soit le sous-corpus. That déterminant est plus utilisé à l'oral. Le complétif est plus utilisé à l'écrit qu'à l'oral et son degré d'utilisation est plus grand en anglais général qu'en médical. Enfin, that relatif est plus fréquent dans le domaine spécialisé médical écrit que dans le domaine de l'anglais général à l'oral. Cette observation rejoint celle de 
Biber et alii (1999: 611) qui montrent que le relatif est moins présent en conversation (1 500 par million de mots) que dans les écrits scientifiques et universitaires (2 300 par million de mots). Ceci peut s'expliquer par le fait que l'écrit scientifique et universitaire, proche du sous-corpus médical écrit, fait plus fréquemment appel à l'hypotaxe. Ce niveau de complexité syntaxique permet l'ajout d'informations pour le nom auquel renvoie that. Le concept en question reçoit des précisions concernant ses caractéristiques ou propriétés, ce qui est courant dans le discours écrit scientifique et universitaire.

Une dernière observation détaillée - toujours à partir des profils par lignes - concerne l'usage de la pro-forme par rapport à celui de la forme déterminante. L'objet d'une telle observation est de mesurer l'écart global entre les deux réalisations fonctionnelles. Il s'agit de voir si ces deux types de fonctionnement ont une régularité, ou bien si des spécificités liées à des corpus apparaissent. La somme des réalisations pour this et pour that permet de construire deux courbes et de constater la part relative de chaque catégorie grammaticale en fonction des sous-corpus (figure 4). Deux observations peuvent être faites. Premièrement, si on prend en compte le sous-corpus médical, celuici favorise légèrement les pro-formes à l'écrit, l'usage étant équivalent à l'oral. À l'inverse, les autres types de sous-corpus démontrent une variation de traitement entre les deux catégories, selon le mode oral ou écrit des sous-corpus. Les deux sous-corpus favorisent le déterminant à l'écrit, mais pas à l'oral. Deuxièmement, l'observation du mode oral révèle une utilisation équivalente des déterminants et pro-formes dans le domaine médical ainsi que dans le domaine scientifique et technologique, mais une proportion plus forte de la pro-forme pour l'anglais général. Cet équilibre entre réalisation fonctionnelle déterminante et pro-forme peut provenir du type de discours. S'agissant de leçons ou de démonstrations, les situations expérimentales impliquent la mention des noms pour faire référence à des entités que le locuteur montre. Il s'agit de l'exophore telle qu'elle a été définie par Halliday et Hasan (1976:33). Dans ce type de situations, l'acte de monstration joue pleinement, en même temps que l'acte de dénomination. À l'inverse, la conversation en anglais général implique moins de dénomination. Une fois construit discursivement, un concept n'a plus besoin d'être nommé. Une reprise anaphorique par la pro-forme suffit pour maintenir la conversation. À titre de comparaison, Biber et alii (1999: 350) ont montré que l'usage de that en conversation est fait en pro-forme dans $70 \%$ des cas, contre $15 \%$ en déterminant. Si on observe le domaine de l'anglais général à l'oral, on note aussi que l'usage pro-forme domine, mais pas dans les mêmes proportions, sachant que cette mesure inclut l'usage de this. 
Figure 4 Pourcentage de pro-formes et de déterminants en fonction des sous-corpus

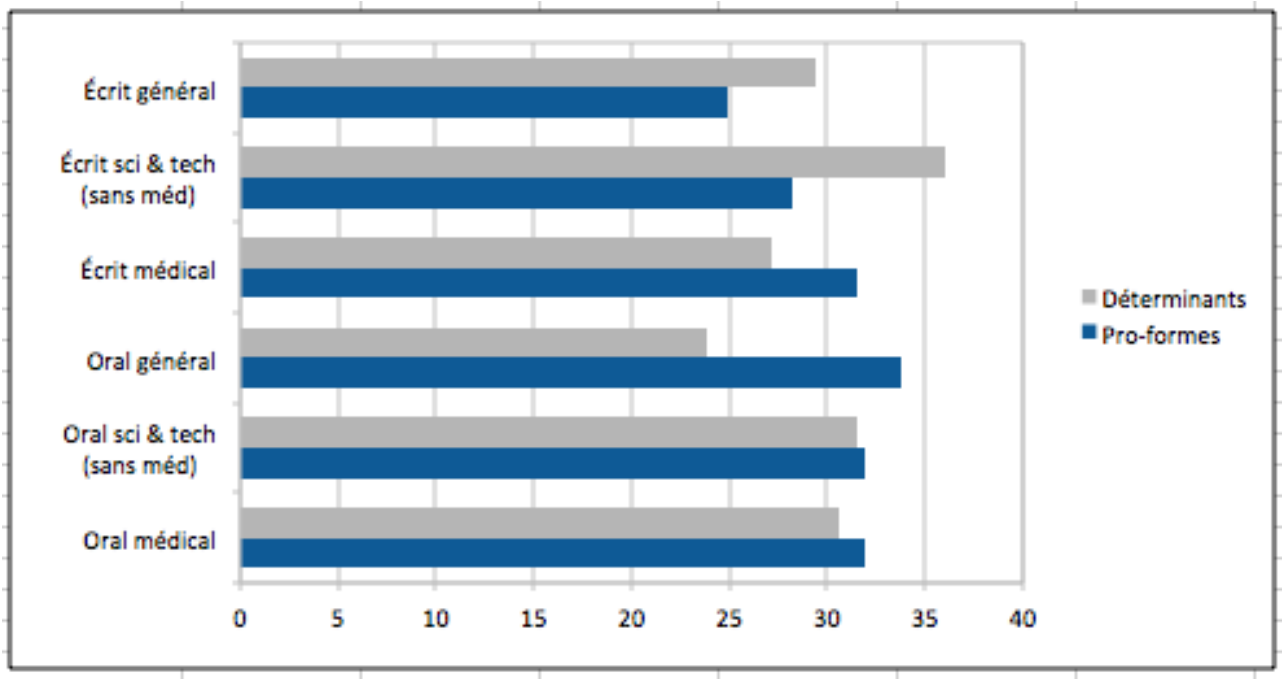

\section{3. Évaluation statistique de la distribution}

Abordons la question de l'indépendance des catégories de données du tableau servant à l'analyse. Il s'agit de savoir si la totalité des observations classées dans des catégories ont une variation aléatoire ou bien si elles sont causées par les catégories $\mathrm{X}$ et $\mathrm{Y}$ du tableau. En d'autres termes, la variation des fréquences d'une catégorie grammaticale de that ou de this est-elle liée à la spécificité du sous-corpus ? Le test du $\chi^{2}$ permet de répondre à cette question. Le test a été effectué sur les données détaillées brutes (1) et normalisées (2) des formes grammaticales de this et de that en fonction des six souscorpus (cf. tableaux 3 et 7). Il s'agit d'un calcul effectué sur l'ensemble des données. Son résultat mesure la différence entre les valeurs attendues et observées et compare cette différence avec ce qui serait attendu en cas de distribution due au hasard. Les résultats suivants sont obtenus :

$\mathrm{X}$-squared $=2610.465, \mathrm{df}=30, \mathrm{p}$-value $<2.2 \mathrm{e}-16$. Without Yates' correction

$\mathrm{X}$-squared $=16532.18, \mathrm{df}=30, \mathrm{p}$-value $<2.2 \mathrm{e}-16$. Without Yates' correction

Le résultat $\mathrm{du} \chi^{2}$ montre une corrélation entre les catégories sous-corpus et les catégories grammaticales de this et de that. La probabilité de l'hypothèse nulle (le fait que les deux catégories soient totalement indépendantes l'une de l'autre et que les fréquences relevées ne soient que le fruit du hasard), mesurée par l'indicateur $p$-value, est très largement inférieure au seuil conventionnel de 0,05 (Gard 2008 inter alia). Le résultat du test semble indiquer un lien entre les deux catégories observées. Cependant, il ne renseigne pas sur l'ampleur de la différence entre les données du tableau. Pour avoir une idée de cette ampleur, il est nécessaire d'utiliser un test qui permet de voir dans quelle mesure l'information contenue dans une colonne contribue au résultat. 
Tableau 7 Données brutes des formes dans le corpus ICE-GB pour le calcul du $\chi^{2}$ (this adverbial est éliminé car $\mathrm{n}<5$ )

\begin{tabular}{|l|c|c|c|c|c|c|c|c|}
\hline \multicolumn{1}{|c|}{ Discours } & $\begin{array}{c}\text { Taille de sous } \\
\text { corpus }\end{array}$ & this pro-forme & $\begin{array}{c}\text { this } \\
\text { déterminant }\end{array}$ & $\begin{array}{c}\text { that pro- } \\
\text { forme }\end{array}$ & $\begin{array}{c}\text { that } \\
\text { déterminant }\end{array}$ & $\begin{array}{c}\text { that } \\
\text { adverbial }\end{array}$ & $\begin{array}{c}\text { that } \\
\text { complétif }\end{array}$ & $\begin{array}{c}\text { that pron } \\
\text { rel }\end{array}$ \\
\hline Oral médical & 15178 & 47 & 103 & 126 & 63 & 39 & 91 & 73 \\
\hline Oral sci \& tech (sans méd) & 20680 & 102 & 171 & 142 & 70 & 51 & 110 & 118 \\
\hline Oral général & 637682 & 1750 & 2875 & 4949 & 1846 & 1451 & 4316 & 2617 \\
\hline Ecrit médical & 8431 & 35 & 43 & 23 & 7 & 0 & 45 & 31 \\
\hline Ecrit sci \& tech (sans méd) & 38202 & 137 & 202 & 52 & 39 & 0 & 176 & 63 \\
\hline Ecrit général & 423581 & 1198 & 1816 & 677 & 406 & 8 & 2630 & 811 \\
\hline
\end{tabular}

La mesure de Cramér V permet d'avoir des informations sur la contribution moyenne des lignes et des colonnes du tableau (Gard 2008). Pour Gard, le test de Cramér permet de réduire le rôle joué par l'impact de la taille de l'échantillon ${ }^{4}$. Le calcul s'effectue sur les données normalisées grâce à l'équation suivante sous $\mathrm{R}^{5}$ :

$$
V=\sqrt{\chi^{2} \div\left(n^{*}(k-1)\right)}
$$

où $\mathrm{n}$ correspond à la somme totale des cellules du tableau et $\mathrm{k}$ correspond au plus petit nombre de colonnes ou de lignes. Sachant qu'un résultat de $100 \%$ représenterait une association parfaite, le résultat obtenu ici montre une association relativement faible (14\%). La combinaison des mesures du $\chi^{2}$ et du V de Cramér permet d'appréhender la corrélation des données croisées. Si le test d'indépendance du $\chi^{2}$ montre une corrélation entre les variables des sous-corpus et les variables relatives aux formes, le V de Cramér permet de prendre la mesure de la contribution moyenne des variables au résultat. Il s'agit ici d'un calcul prenant en compte l'ensemble des facteurs dans leur globalité et il est intéressant de mesurer les contributions individuelles aux résultats du $\chi^{2}$. Cornillon et alii $(2010: 109)$ décrivent la procédure à suivre sous $\mathrm{R}$ avec la formule suivante :

round $\left(100^{*}\right.$ cthisthat\$residuals ${ }^{\wedge}$ / cthisthat $\$$ stat, 1$)$

qui permet d'isoler les composantes individuelles du calcul du $\chi^{2}$.

On peut étudier plus en détail cette liaison (avec le $\chi^{2}$ ) en calculant les contributions

à la statistique $\chi^{2}$ observée. Les racines carrées des contributions sont dans l'objet residuals (du logiciel R). En divisant chaque terme par le total (i.e., la valeur de $\chi^{2}$ observée contenue dans la composante stat), on obtient un pourcentage. (Cornillon et alii 2010)

Le tableau 8 fait la synthèse des contributions individuelles. Globalement, on trouve deux groupes de résultats. Le premier groupe concerne ceux qui contribuent faiblement au $\chi^{2}$, par exemple this déterminant à l'oral médical. Le second groupe concerne les combinaisons qui contribuent modérément à la non-indépendance des deux groupes de variables. Par exemple, that complétif est un levier important dans l'anglais écrit général.

Tableau 8 Calcul des contributions au résultat

\begin{tabular}{|l|c|c|c|c|c|c|c|}
\hline \multicolumn{1}{|c|}{ Discours } & $\begin{array}{c}\text { This pro- } \\
\text { forme }\end{array}$ & $\begin{array}{c}\text { This } \\
\text { déterminant }\end{array}$ & $\begin{array}{c}\text { That pro- } \\
\text { forme }\end{array}$ & $\begin{array}{c}\text { That } \\
\text { déterminant }\end{array}$ & $\begin{array}{c}\text { That } \\
\text { adverbial }\end{array}$ & $\begin{array}{c}\text { That } \\
\text { complétif }\end{array}$ & $\begin{array}{c}\text { That pron } \\
\text { rel }\end{array}$ \\
\hline Oral médical & 3,4 & 0,5 & 3,6 & 3 & 3,3 & 2 & 0 \\
\hline Oral sci \& tech (sans méd) & 0 & 0,1 & 0,1 & 0,2 & 2,2 & 5 & 0,6 \\
\hline Oral général & 2,8 & 4 & 5,5 & 0,3 & 3,2 & 0 & 0 \\
\hline Ecrit médical & 3,3 & 0,3 & 2,1 & 3,1 & 6 & 0,6 & 1 \\
\hline Ecrit sci \& tech (sans méd) & 4,2 & 4 & 6 & 0,7 & 4,8 & 1,3 & 1,4 \\
\hline Ecrit général & 0,6 & 0,4 & 4,7 & 1,1 & 4,7 & 9,3 & 0,6 \\
\hline
\end{tabular}

Toujours selon Cornillon et alii (2010), « un retour aux données, via les résidus et, notamment, leurs signes ", permet de voir quelles combinaisons favorisent l'hypothèse 
d'indépendance et avec quelle importance. Une valeur 0 indique une combinaison correspondant à une distribution attendue. Par exemple, les données du tableau 9 permettent de dire que that complétif est plus important qu'attendu à l'écrit. Inversement, le nombre d'occurrences de that en fonction adverbiale est beaucoup plus faible qu'attendu (statistiquement), en raison de sa très faible utilisation à l'écrit. On retrouve la très forte contribution du that pro-forme à l'anglais général et médical à l'oral. Sa contribution au sous-corpus science et technologie est nulle par rapport à ce qui serait attendu si l'hypothèse d'indépendance était vérifiée. De la même manière, this pro-forme est plus significatif à l'écrit dans le domaine médical et dans le domaine scientifique et technologique. This déterminant prend une plus grande place dans les productions écrites scientifique et technologique, et une place beaucoup plus négligeable dans les productions relevant de l'oral général.

Tableau 9 Mesure de l'écart entre les contributions réelles et celles fondées sur l'hypothèse d'indépendance

\begin{tabular}{|l|c|c|c|c|c|c|c|}
\hline \multicolumn{1}{|c|}{ Discours } & $\begin{array}{c}\text { This pro- } \\
\text { forme }\end{array}$ & $\begin{array}{c}\text { This } \\
\text { déterminant }\end{array}$ & $\begin{array}{c}\text { That pro- } \\
\text { forme }\end{array}$ & $\begin{array}{c}\text { That } \\
\text { déterminant }\end{array}$ & $\begin{array}{c}\text { That } \\
\text { adverbial }\end{array}$ & $\begin{array}{c}\text { That } \\
\text { complétif }\end{array}$ & $\begin{array}{c}\text { That pron } \\
\text { rel }\end{array}$ \\
\hline Oral médical & $-23,829$ & $-9,349$ & 24,443 & 22,35 & 23,37 & $-18,403$ & $-0,619$ \\
\hline Oral sci \& tech (sans méd) & 0,448 & 4,573 & 3,638 & 6,242 & 19,073 & $-28,688$ & 9,671 \\
\hline Oral général & $-21,432$ & $-25,845$ & 30,087 & 6,702 & 22,857 & 1,947 & $-1,794$ \\
\hline Ecrit médical & 23,348 & 6,676 & $-18,524$ & $-22,798$ & $-31,534$ & 10,16 & 13,06 \\
\hline Ecrit sci \& tech (sans méd) & 26,206 & 25,561 & $-31,43$ & $-11,072$ & $-28,249$ & 14,413 & $-14,982$ \\
\hline Ecrit général & 9,565 & 8,044 & $-27,907$ & $-13,273$ & $-27,824$ & 39,264 & $-10,283$ \\
\hline
\end{tabular}

Cette approche a permis un retour aux données. Les données du tableau montrent les résidus, c'est-à-dire les différences entre les fréquences relatives et les fréquences attendues. La section 4 poursuit cette étude des données en proposant l'analyse de textes provenant des deux sous-corpus médicaux oral et écrit.

\section{Analyse de la distribution textuelle}

Les analyses précédentes étaient centrées sur une approche statistique dont l'objectif était d'observer des tendances tout en assurant la validité de la distribution des données. Cette section consiste à effectuer un retour qualitatif sur certaines des données qui composent les sous-corpus médicaux oral et écrit.

À partir de l'interface ICECUP ont été extraits quatre textes oraux (5 200 mots) et quatre textes écrits (9000 mots). Ces textes ont été importés dans le concordancier AntConc ${ }^{6}$ qui permet d'effectuer plusieurs types de calculs, dont les fréquences des formes et les fréquences de clusters, c'est-à-dire les fréquences de $n$-grams (séquence contiguë de $\mathrm{n}$ mots) incluant une forme pivot. En outre, on peut visualiser la distribution textuelle d'une forme et savoir si on la trouve de manière régulière tout au long d'un texte ou plus regroupée en début, milieu ou fin de texte.

41 Si on s'intéresse à l'oral médical, et plus particulièrement aux clusters 2-grams incluant that, les plus fréquents sont that's ( 48 occurrences) et than that (10 occurrences). Il s'agit de l'usage de that en pro-forme, ce qui corrobore les observations faites plus haut. Pour this, le nombre d'occurrences dans les quatre textes est trop faible pour extraire des fréquences significatives, même si la pro-forme ressort statistiquement avec this is (2 occurrences). Il aurait été intéressant de procéder de la sorte avec des textes de l'anglais général afin de faire ressortir les cluster 2-grams les plus fréquents et ainsi de comparer avec le domaine médical. Cependant, cette partie qualitative se limite à la distribution textuelle dans les textes médicaux. La sélection de seulement quelques 
textes de l'anglais général s'appliquerait mécaniquement sur des champs particuliers, ce qui contredirait le concept même d'anglais général.

La mise en regard des graphes de distribution permet d'appréhender la distribution textuelle de la forme that. La figure 5 montre que, selon le locuteur, la densité textuelle à l'oral est variable. On note toutefois l'existence d'intervalles sans la forme après des périodes de densité forte. L'explication réside peut-être dans l'alternance de phases transitoires entre thématiques, avec des phases explicatives. Lors de phases explicatives ou descriptives dans le domaine médical, le locuteur est amené à devoir reprendre l'objet réel ou abstrait présenté dans son discours. Il tend donc à utiliser la pro-forme pour la mise en œuvre référentielle. D'autre part, le locuteur doit aussi expliciter des concepts et that complétif est adapté dans ce cas puisqu'il permet la subordination par rapport à des propositions englobantes telles que you mean that. L'étude statistique a montré que la pro-forme et le complétif étaient prépondérants dans le domaine médical à l'oral.

Figure 5 Distribution textuelle de that dans des textes oraux médicaux

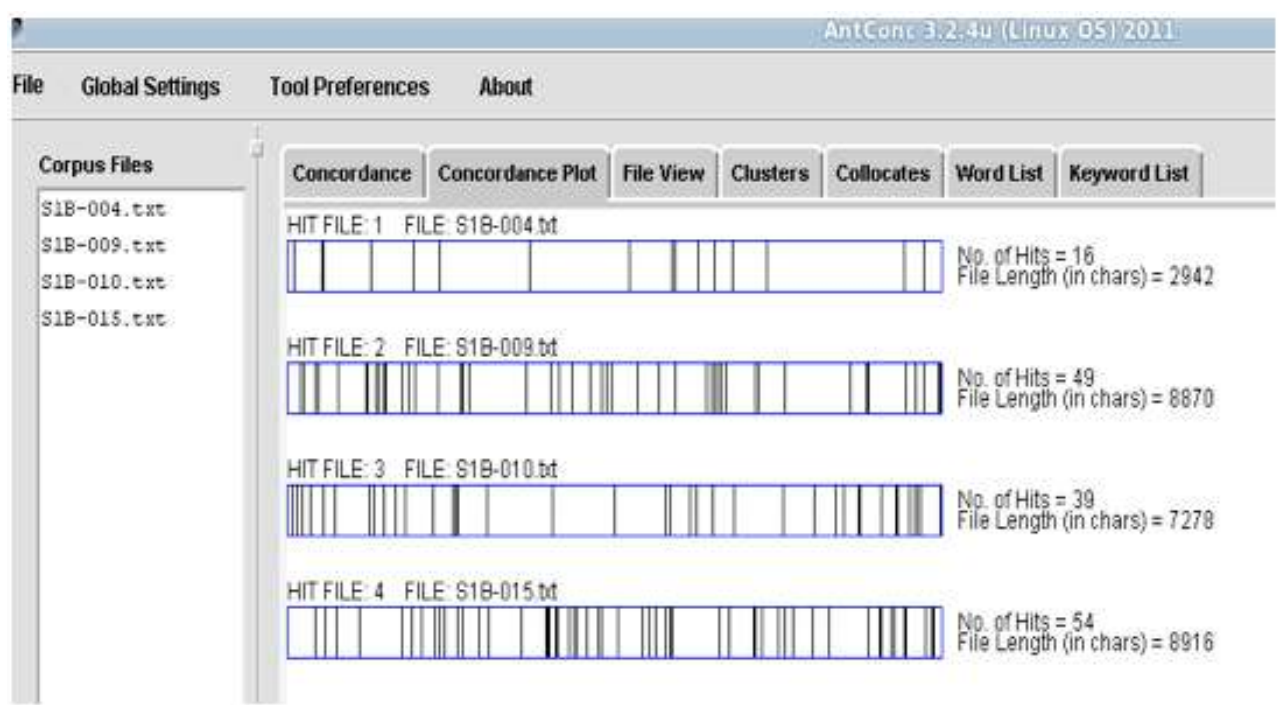

L'étude de l'écrit médical suit la même procédure. Les clusters les plus fréquents pour that sont that the (11), that is/are (7) et that you (5). Ce résultat correspond aux résultats statistiques montrant un usage important des that complétifs et relatifs au sein du souscorpus médical. Pour this, les plus fréquents sont this is (7) et this stage (4). L'étude statistique montre une légère supériorité de this en pro-forme au sein du sous-corpus médical écrit et, malgré les faibles fréquences, on retrouve cette tendance dans l'échantillon. L'étude de la distribution textuelle de that dans des textes écrits médicaux (cf. figure 6) montre des écarts prononcés dans les usages. À l'écrit, le « which " concurrence la forme relative. Dans ce mode, la nécessité de reprise par le biais du that pro-forme semblerait moins forte. L'écrit médical appelle un usage endophorique de that. Or, les articles médicaux impliquent des développements cherchant à décrire et à mettre des concepts au premier plan, ce qui s'oppose à la valeur de rejet de that (Fraser \& Joly, $1980: 38$ ). On retrouve aussi les phases de silence par rapport aux phases d'utilisation dense. Il y a moins d'opportunités d'utilisation du that dans le domaine médical écrit mais, lorsque celui-ci apparaît, les mécanismes de référence et de complexité syntaxique, avec le complétif ou relatif, sont pleinement exploités. 
Figure 6 Distribution textuelle de that dans des textes écrits médicaux

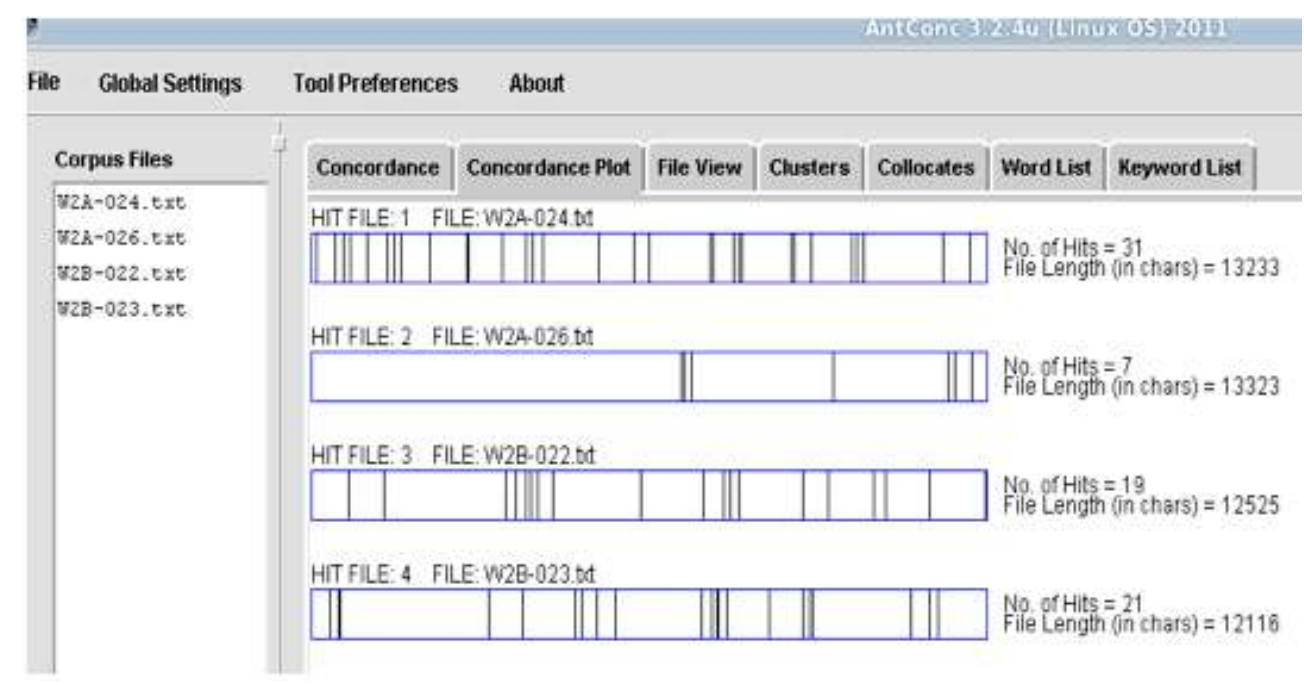

\section{Conclusion}

L'étude comparative globale révèle une différence d'usage entre this et that, que le domaine soit spécialisé ou non, et quelle que soit la spécialité. This est utilisé de manière équivalente à l'oral et à l'écrit, that prédomine à l'oral. Cependant, la présente étude, fondée sur la fonction grammaticale, montre que this et that ont un comportement spécifique dans les domaines spécialisés scientifiques. En effet, l'analyse détaillée des formes selon leur catégorie grammaticale montre une hétérogénéité au sein de l'apparente stabilité de this. Celui-ci se retrouve plus dans les textes scientifiques qu'en anglais général. This pro-forme est plus fréquent dans le mode mode écrit qu'à l'oral dans le domaine médical, par exemple. L'analyse détaillée de that révèle son rôle important en pro-forme à l'écrit, quel que soit le domaine, mais pas à l'oral. Le complétif suit une tendance inverse.

Le test d'indépendance des données effectué avec le calcul du $\chi^{2}$ montre une dépendance de faible niveau entre les sous-corpus et les constituants grammaticaux. Ce résultat global faible cache des variations importantes lors de l'analyse des contributions individuelles à ce résultat. Celles-ci montrent que certaines formes comme le complétif verbal ont un rôle plus important que statistiquement attendu. La dernière partie de l'étude propose un retour sur les données en explorant la distribution textuelle d'un échantillon de textes oraux et écrits du domaine médical. Les rôles de certains constituants grammaticaux entrent en résonance avec l'étude statistique.

Cette étude contribue à l'effort de recherche visant à mettre au jour le rôle des différents constituants grammaticaux en fonction des discours. La démarche se positionne entre l'étude de la morphosyntaxe et des divers sens de marqueurs. Elle se fonde sur l'observation de l'évolution d'une forme en fonction de variations liées au contexte, donc au sens, et sur une analyse fonctionnelle des formes et de leurs caractéristiques morphosyntaxiques. Ce travail contribue de la sorte à l'inventaire des caractéristiques linguistiques propres à certains domaines spécialisés comme les sciences médicales et il peut être articulé à leur enseignement et à leur apprentissage. 
Nous remercions les participants au colloque GERAS pour leurs suggestions et remarques. Nos remerciements s'adressent aussi à Nicolas Ballier de l'Université Paris-Diderot, à Pascale Sébillot de l'IRISA/INSA de Rennes pour leur relecture et recommandations et à Geneviève Bordet de l'Université Paris-Diderot pour ses commentaires pertinents.

\section{BIBLIOGRAPHIE}

Aarts, Bas, Gerald Nelson et Sean Wallis. 2007. « Using fuzzy tree fragments to explore English grammar ». English Today 23/2, 27-31.

Biber, Douglas, Stig Johanson, Geoffrey Leech, Susan Conrad et Edward Finegan. 1999. Longman Grammar of Spoken and Written English. Harlow : Longman.

Bordet, Geneviève. 2011. «This comme marqueur privilégié du genre : le cas des résumés de thèses ». Discours 9 [En ligne]. Consulté le 25 mai 2013. <http://discours.revues.org/8506>.

Carter-Thomas, Shirley et Elizabeth Rowley-Jolivet. 2001. « Syntactic differences in oral and written scientific discourse: the role of information structure ». ASp [En ligne] 31-33.

Consultation le 25 mai 2013. <http://asp.revues.org/1752>.

Cornillon, Pierre André, Arnaud Guyader, François Husson, Nicolas Jégou, Julie Josse, Maela Kloareg, Eric Matzner-Lober et Laurent Rouvière. 2010. Statistiques avec R. $2^{\mathrm{e}}$ édition augmentée. Rennes : Presses Universitaires de Rennes.

Cornish, Francis. 1999. Anaphora, Discourse, and Understanding. Evidence from English and French. Oxford : Oxford University Press.

Cotte, Pierre. 1993. «De l'étymologie à l'énonciation ». Travaux de linguistique et de philologie 31, 43-89.

Fraser, Thomas et André Joly. 1979. « Le système de la deixis - Esquisse d'une théorie d'expression en anglais ». Modèles Linguistiques 1/2, 97-157.

Fraser, Thomas et André Joly. 1980. « Le système de la deixis (2) : endophore et cohésion discursive en anglais ». Modèles Linguistiques 2/2, 22-51.

Gard, B. Jenset. 2008. « Basic statistics for corpus linguistics ». Université de Bergen. Consulté le 25 mai 2013. <http://folk.uib.no/gje037/statTutorialR.pdf>.

Gaillat, Thomas. 2013. « This and that in native and learner English: From typology of use to tagset characterisation ». In S. Granger, G. Gilquin et F. Meunier (dir.), Twenty Years of Learner Corpus Research: Looking back, Moving ahead. Corpora and Language in Use - Proceedings 1. Louvain-laNeuve : Presses universitaires de Louvain.

Gries, Stefan Th. 2010. « Useful statistics for corpus linguistics ». In Aquilino S. et A. Moisés (dir.), A Mosaic of Corpus Linguistics: Selected Approaches. Peter Lang : Francfort-sur-le-Main, 269-291.

Halliday, Michael A. K. et Ruqaiya Hasan. 1976. Cohesion in English. English Language Series. Harlow : Pearson Education Limited. 
Huddleston, Rodney et Geoffrey K. Pullum. 2002. The Cambridge Grammar of the English Language. Beccles, Suffolk : Cambridge University Press.

Hyland, Ken. 2010. «Constructing proximity: Relating to readers in popular and professional science ». Journal of English for Academic Purposes 9/2, 116-127.

Kleiber, Georges. 1992. « Anaphore-deixis : deux approches concurrentes ». In Morel, M.-A. et L. Danon-Boileau, La Deixis. Paris : Presses Universitaires de France, 613-626.

Lapaire, Jean-Rémi et Wilfrid Rotgé. 1998. Linguistique et grammaire de l'anglais. Toulouse : Presses Universitaires du Mirail.

Nelson, Gerald, Sean Wallis et Bas Aarts. 1998. « The British component of the International Corpus of English (ICE-GB) and ICECUP Software (CD-ROM) ». Londres. Consulté le 25 mai 2013. <http://www.ucl.ac.uk/english-usage/projects/ice-gb/>.

Nelson, Gerald, Sean Wallis et Bas Aarts. 2002. Exploring natural language: Working with the British component of the International Corpus of English. Amsterdam : John Benjamins Publishing Co.

Parkinson, Jean et Ralph Adendorff. 2004. «The use of popular science articles in teaching scientific literacy ». English for Specific Purposes 23/4, 379-396.

Petit, Michel. 2010. « Le discours spécialisé et le spécialisé du discours : repères pour l'analyse du discours en anglais de spécialité ». E-rea [En ligne] 8/1. Consulté le 25 mai 2013. <http:// erea.revues.org/1400>.

Quirk, Randolph, Geoffrey Leech et Jan Svartvik. 1985. A Grammar of Contemporary English. Londres, Beccles et Colchester : Longman.

\section{NOTES}

1. Le chapitre a été écrit par Lesley Stirling et Rodney Huddleston.

2. <http://www.ucl.ac.uk/english-usage/resources/icecup/index.htm>. Consulté le 6 mai 2013.

3. L'accès aux fonctions, catégories et traits (Features) se fait à l'adresse suivante <http:// www.ucl.ac.uk/english-usage/resources/grammar/index.htm>. Consulté le 6 mai 2013.

4. «Cramér V 'factors out' the size of the sample, and gives the 'average' contribution of rows and columns (that is, the categories in the table and their respective observations in the rows and columns) to the final result ».

5. Formule sous $\mathrm{R}$ : $\quad \mathrm{cV}<-$-sqrt(chisq.test(thisthatnrmzd,correct=FALSE)\$statistic/ (sum(thisthat)* $\min (\operatorname{dim}($ thisthat)-1))) et résultat : $\mathrm{X}$-squared $=0.1433692$. Explication donnée sur <http://www.biostat.wustl.edu/archives/html/s-news/2003-09/msg00185.html>. Consulté le 24 mai 2013.

6. <http://www.antlab.sci.waseda.ac.jp/antconc_index.html>. Consulté le 24 mai 2013.

\section{RÉSUMÉS}

Cet article analyse les caractéristiques distributionnelles des deux démonstratifs « this » et " that » afin d'identifier des usages spécifiques en fonction de domaines spécialisés de l'anglais. Les 
données sont collectées dans le corpus ICE-GB. L'étude consiste à échantillonner le corpus en sous-corpus, en fonction du domaine spécialisé et du mode écrit ou oral des textes. Les souscorpus relevant de l'anglais général sont distingués de ceux relevant de domaines spécialisés (médecine, science et technologie). Pour chaque sous-corpus, l'outil ICECUP est utilisé pour effectuer des requêtes et extraire le nombre d'occurrences des démonstratifs en fonction de leur catégorie grammaticale: déterminant, pro-forme, adverbe, complétif et pronom relatif. La distinction par catégorie vise à compter le nombre exact d'une forme particulière en fonction d'un sous-corpus spécifique représentant un domaine. Les résultats statistiques globaux montrent une corrélation limitée entre les démonstratifs et les domaines spécialisés. Cependant, certaines catégories grammaticales entretiennent un lien étroit avec un domaine particulier. Enfin, l'étude montre des tendances marquées concernant l'usage des démonstratifs dans leur rôle de pro-forme.

This paper analyzes the distributional characteristics of demonstratives "this" and "that" so as to identify specific uses in relation to specialized domains of English. Data were collected from the ICE-GB corpus. The study consisted in extracting - oral or written - samples from sub-corpora linked to a specialized domain. The author classified the sub-corpora according to whether they corresponded to general English or to the medical, scientific and technological domains. For each sub-corpus, ICECUP was used to make queries and extract the number of occurrences of the demonstratives according to their syntactic function. Grammatical function (i.e., determiner, pro-form, adverb, complementizer or relative pronoun) was used to establish exact counts of a particular form in relation to a specific sub-corpus representing a specialized domain. The statistical results show a limited correlation between the demonstratives and the domains at a global level, but some specific functions do have a substantial link with particular specialized domains. The study reveals marked trends regarding the use of demonstratives in their pro-form function.

\section{INDEX}

Mots-clés : démonstratif, déterminant, domaine spécialisé, pro-forme, that, this Keywords : demonstrative, determiner, pro-form, specialized domain, that, this

\section{AUTEUR}

\section{THOMAS GAILLAT}

Thomas Gaillat, en poste à l'Université Rennes 1, est enseignant d'anglais depuis 1999 ; il est membre des laboratoires CLILLAC-ARP et LIDILE. Il prépare une thèse à l'Université Paris Diderot au sujet de l'interopérabilité des corpus d'apprenants en anglais ; ses recherches portent plus particulièrement sur l'annotation automatique des démonstratifs this et that dans les corpus d'apprenants. thomas.gaillat@univ-rennes1.fr 